\title{
Facile approach to the synthesis of molecularly imprinted ratiometric fluorescence nanosensor for the visual detection of folic acid
}

\author{
Chuyao Li ${ }^{\mathrm{a}, \mathrm{b}}$, Qian Yang ${ }^{\mathrm{a}, \mathrm{b}}$, Xiaoyan Wang ${ }^{\mathrm{b}}$, Maryam Arabi ${ }^{\mathrm{b}}$, Hailong Peng ${ }^{\mathrm{a}, \mathrm{c}}$, Jinhua $\mathrm{Li}^{\mathrm{b}}$, \\ Hua Xiong ${ }^{\mathrm{a}, *}$, Lingxin Chen ${ }^{\mathrm{b}, \mathrm{d}, *}$ \\ a State Key Laboratory of Food Science and Technology, Nanchang University, Nanchang 330047, China \\ ${ }^{\mathrm{b}}$ CAS Key Laboratory of Coastal Environmental Processes and Ecological Remediation, Research Center for Coastal Environmental Engineering and Technology, Yantai \\ Institute of Coastal Zone Research, Chinese Academy of Sciences, Yantai 264003, China \\ ${ }^{\mathrm{c}}$ School of Resources, Environmental, and Chemical Engineering, Nanchang University, Nanchang 330031, China \\ ${ }^{\mathrm{d}}$ College of Chemistry and Chemical Engineering, Qufu Normal University, Qufu 273165, China
}

A R T I C L E I N F O

\section{Keywords:}

Ratiometric fluorescence

Molecular imprinting

Visual detection

Folic acid

\begin{abstract}
A B S T R A C T
A core-shell molecular imprinting fluorescence nanosensor was developed for the ratiometric fluorescence and visual detection of folic acid (FA). The nanosensor was prepared by anchoring imprinting shell on the silica nanoparticles, and embedding the CdTe quantum dots in imprinted shell to provide FA-dependent fluorescence signals. Under the optimum conditions, a favorable linearity relationship between the fluorescence intensities ratio $\left(\mathrm{I}_{449} / \mathrm{I}_{619}\right)$ and the FA concentration over $0.23-113 \mu \mathrm{M}$ was offered with a detection limit (LOD) of $48 \mathrm{nM}$. The visual detection for FA was realized by evaluating profuse fluorescence color change from red to pink to purple to final blue. The proposed sensor possessed excellent sensing performances of rapid response, high precision, super sensitivity and selective recognition. Furthermore, endogenous FA was detected in real samples ranging from 37.4 to $265.8 \mu \mathrm{g} / 100 \mathrm{~g}$; satisfactory spiked recoveries were obtained within $94.8-104.2 \%$, which conformed to the measurement results by HPLC-UV.
\end{abstract}

\section{Introduction}

Molecular imprinting technology is a promising method for to produce artificial receptors with tailor-made binding sites in a strict polymer matrix, namely molecularly imprinted polymers (MIPs). These binding sites are complementary to the targeted analytes in size, shape, and functional groups, so to possess specific recognition ability and improve the selectivity and the detection efficacy (Komasawa, Ueki, Kaminoh, \& Nishi, 2010). Besides the high affinity to targets, other unique superiorities of MIPs, namely good chemical stability, physical robustness, easy preparation and low cost, have been spotlighted to be an ideal alternative to biological receptor (i.e., antibodies and enzymes) in many fields, including sample pretreatment (Chen, Xu, \& Li, 2011), immunoassays (Chen et al., 2018), biomimetic catalysis (Menger et al., 2016) and so on. Especially, in biological/chemical sensing field (Yang et al., 2018), MIPs have been turned out to be a hotspot.

Sensors can output measurable signals via various transduction mechanisms, including electrical, optical, magnetic, thermal and others
(Wang et al., 2018). Currently, fluorescence sensors have aroused great research interest in detection application by their apparent advantages of high sensitivity, simplicity, and short incubation time (Wang et al., 2017). Thus, in combination with the fantastic selectivity of MIPs and splendid sensitivity of fluorescence sensors, it is naturally available to design MIPs based fluorescence sensors to obtain superior sensing performances. Among many fluorescent materials, semiconductor quantum dots (QDs) are enthusiastically pursued owing to their bright photoluminescence, size-dependent narrow emission peak, larger Stokes shift, good photostability etc., especially in comparison to organic dyes. Recent years have witnessed rapidly increasing interest in QD-modified MIPs sensors for ions, small molecules, and even biomacromolecules (Qi et al., 2017; Wu et al., 2017; Yang, He, Wang, Li, \& Zhang, 2014). Generally speaking, ratiometric fluorescence sensors with self-calibration capability are less susceptible to analyte-independent factors than that of "single-irradiation" mode, and thereupon enhance precision and sensitivity of the analytical method ( $\mathrm{Li}$ et al., 2018). Moreover, depending on the variation of fluorescence

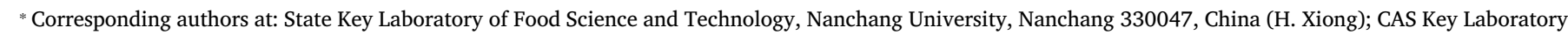

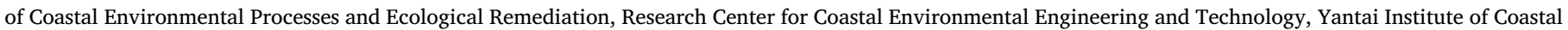
Zone Research, Chinese Academy of Sciences, Yantai 264003, China (L. Chen).

E-mail addresses: huaxiong100@126.com (H. Xiong), lxchen@yic.ac.cn (L. Chen). 
intensity ratio, ratiometric fluorescence sensors can acquire profuse color evolution rather than the merely brightness change, realizing the naked-eye determination for targeted analytes ( Fu, Jin, Bu, \& Gui, 2018). Hence, an increasing number of the ratiometric fluorescence MIPs sensors have been proposed for various targets.

Traditionally, the ratiometric fluorescence MIPs sensors are prepared by incorporating two different fluorescent substances into imprinting matrix via one-pot (Li et al., 2016). The deeply-located imprinted cavities in the highly-crosslinked MIPs vastly restrain site accessibility and mass transfer. What's worse, the fluorescent substances are often distributed unevenly so that the imprinted cavities are completely not surrounded by fluorescent substances and the rebinding behavior of trace targets cannot induce signal change, weakening the sensitivity of sensors (Xu \& Lu, 2015). Hence, the surface imprinting strategy was brought in to propose the core-shell structured ratiometric fluorescence MIPs. Briefly, the reference dye is encapsulated into the supporting cores firstly, and then the imprinted shell is anchored on the core surface in the presence of target-sensitive fluorescence materials (Wang, Yu, Wu, et al., 2016). The ultra-thin imprinting shell possesses lower mass-transfer resistances and provides higher recognition site accessibility, in favor of a rapid response to the targeted analytes (Zhang et al., 2017). Drawing lessons from the time-saving procedure of one-pot methods, we aimed to build the core-shell structured ratiometric fluorescence MIPs sensors by embedding two fluorescence signal providers into MIPs shell simultaneously, for highly selective and sensitive detection of targeted substances in complex samples.

Folic acid (FA), otherwise known as Vitamin B9 in pteroylmonoglutame and/or polyglutamted forms (upper of Fig. S1), is a water-soluble vitamin B-complex group. As a cofactor for enzymes, FA plays an essential role in one carbon metabolism (Pawlosky \& Flanagan, 2001). Its deficiency is associated with cardiovascular exacerbation, malformation, and neural tube defects during pregnancy (Wang, De, et al., 2016). And the excess of FA would interfere with the absorption of vitamin $\mathrm{B}_{12}$ and zinc, so to cause other health problems (Li, GueantRodriguez, et al., 2017). Human are unable to synthesize folic acid and could only gain it from foodstuffs and fortified foods. Green vegetables, yeast and some fruits (citrus) are a good dietary source of FA (Delchier, Herbig, Rychlik, \& Renard, 2016). Meanwhile, many fortified foods are nowadays commercially available. Nevertheless, there is a relatively low margin of safety between adequate levels of intake and maximum safe intake, it is necessary to monitor the levels that can be added safely to foods (Leporati et al., 2005). Unfortunately, analysis of FA is not an easy task because of its instability under acidic conditions and sensitivity to light and heat. Conventional analytical techniques employed for the FA quantification mainly include high performance liquid chromatography-mass spectrometry (HPLC-MS) (Monica, Alain, Elodie, \& Laurent, 2014), LC-MS/MS (Vishnumohan, Arcot, \& Pickford, 2011), bioassays, and fluorimetric methods (Hemmateenejad, Shakerizadehshirazi, \& Samari, 2014), which could provide available and reliable test results. It is a pity that they all have the individual drawbacks, for instance, tedious sample pretreatment of HPLC, time-consuming detection of bioassays, difficult design of fluorimetric method. Importantly, all the above methods almost have failed to realize the visual detection of the target. Accordingly, the development of facile, rapid and cost-effective methods remains necessary while providing the same sensitivity level combined with a selective detection. To the best of our knowledge, there are no reports with reference to ratiometric fluorescence sensors based on molecularly imprinted for visual recognition and detection of FA.

Therefore, in this work, we proposed a molecular imprinting ratiometric fluorescence sensor via a facile sol-gel method for the rapid, sensitive and visual identification and detection of FA. The synthesized FA-imprinted shell was anchored on the silica nanoparticles core, where FA, 3-aminopropyltriethoxysilane (APTES), tetraethyloxysilane (TEOS) and cadmium telluride (CdTe) QDs served as model template, functional monomer, cross-linker and analyte-dependent fluorescence signal provider, respectively. Not only was the tedious synthesis route intelligently omitted, but also imprinting sites anchored on the surface of silica matrix enhanced the sensitivity. The as-obtained sensor possessed two separated emission peaks at 449 and $619 \mathrm{~nm}$ under a single excitation wavelength $(365 \mathrm{~nm})$, belonging to FA-trigger blue fluorescence and the red fluorescence of CdTe QDs, respectively. Upon the addition of FA, the MIPs sensor could rebind FA, causing blue fluorescence increase while red fluorescence decrease, followed by a distinct fluorescence color change covering red, pink, purple, and blue. And therefore, based on the corresponding quenching amount, the sensitivity and selectivity quantitative detection of FA was achieved. Finally, the practical application of the developed sensor in six different real samples was investigated, exhibiting high selectivity, simplicity, rapidity, accuracy, and good practical feasibility.

\section{Materials and methods}

\subsection{Materials and reagents}

Folic acid (FA) was purchased from Solarbio (Beijing, China), and methotrexate (MTX), trimethoprim (TMP), cysteine (Cys), arginine (Arg), histidine (His), glutamic acid (Glu), and vitamin C (Vc) were supplied by Sinopharm Chemical Reagent Co. Ltd (Shanghai, China); Fig. S1 shows their chemical structures. Sodium borohydride and tellurium powder were purchased from Sigma-Aldrich (Shanghai, China). 3-Aminopropyltriethoxysilane (APTES), tetraethyloxysilane (TEOS), Cadmium chloride hemi(pentahydrate) $\left(\mathrm{CdCl}_{2} \cdot 2.5 \mathrm{H}_{2} \mathrm{O}\right)$, 3-mercaptopropionic acid (MPA), mercaptoacetic acid (TGA) and glutathione (GSH) were bought from Aladdin (Shanghai, China). Ethanol, methanol, acetonitrile and ammonium solution were supplied by Sinopharm Chemical Reagent Co. Ltd (Shanghai, China). All chemicals used were of analytical grade. All aqueous solutions throughout this work were prepared with ultrapure deionized water of $18.2 \mathrm{M} \Omega$ specific resistance (Millipore, Bedford, MA, USA).

\subsection{Instrumentation}

Fourier transform infrared (FT-IR) spectra were recorded on a spectrophotometer (Nicolet 5700, Thermo Nicolet Corp., USA) using the $\mathrm{KBr}$ plate (at $4000-400 \mathrm{~cm}^{-1}$ ) at the resolution of $4 \mathrm{~cm}^{-1}$. The morphological evaluation was carried out by scanning electron microscope (SEM, JSM-6701F, Japan) and transmission electron microscope (TEM, JEM-2100, Japan). Detailed sample preparation process for SEM and TEM was described in Experimental S1. Fluorescence spectra were obtained on Fluoromax-4 Spectrofluorometer (Horiba Scientific). Absorption spectra were measured by using a UV-Vis Spectrophotometer (Tu 1900, Beijing Purkinje General Instrument Co., Ltd. China). Dynamic light scattering (DLS) analysis was performed on Malvern Zetasizer Nano-ZS90 (ZEN3590, UK). Elemental analysis was carried out via Energy Dispersive Spectrometer (EDS, JSM-6701F, Japan). HPLC-UV was performed on an Agilent 1260 Infinity II system (Agilent Technologies, Germany) equipped with autosampler and diode array detector (DAD), and the test conditions for FA were described in detail in Experimental S2.

\subsection{Synthesis of TGA/MPA/GSH-capped CdTe QDs}

Water-soluble TGA-modified CdTe QDs were prepared by a refluxing route ( $\mathrm{Xu}$ et al., 2013) with slight modification, with a preparation flow chart in Scheme S1. Briefly, $68.4 \mathrm{mg}$ of $\mathrm{CdCl}_{2} \cdot 2.5 \mathrm{H}_{2} \mathrm{O}$ was firstly dissolved in $75 \mathrm{~mL}$ of ultrapure water, followed by adding $63 \mu \mathrm{L}$ of TGA under stirring. Then, the $\mathrm{pH}$ value of the solution was rapidly adjusted to about 9.0 by sodium hydroxide $\left(1 \mathrm{~mol} \mathrm{~L}^{-1}\right)$. Next, $1 \mathrm{~mL}$ of the freshly prepared NaHTe aqueous solution (prepared by using $40 \mathrm{mg}$ of sodium borohydride and $38.3 \mathrm{mg}$ of tellurium powder in $40{ }^{\circ} \mathrm{C}$ for $4 \mathrm{~h}$ ) was injected into the above solution in a nitrogen atmosphere. The 
mixture was refluxed for certain time until the red-emission CdTe QDs were obtained. Meanwhile, MPA- or GSH-capped CdTe QDs were synthesized using the same method but replaced TGA with $78.5 \mu \mathrm{L}$ of MPA or $0.182 \mathrm{~g} \mathrm{GSH}$, respectively.

\subsection{Preparation of molecular imprinting ratiometric fluorescence sensor}

The molecular imprinting ratiometric fluorescence sensor (FL-MIPs sensor, also MIPs for simplicity) was fabricated on the surface of silica nanoparticles via a simple sol-gel method (Wang, Yu, Kang et al., 2016) with necessary modification. Firstly, silica nanoparticles were synthesized as reported (Wu et al., 2015) with details given in Experimental S3 and Scheme S1. Subsequently, $1 \mathrm{~mL}$ of silica aqueous solution and $3 \mathrm{~mL}$ of red-emission CdTe QDs were dispersed in $8 \mathrm{~mL}$ of water. Then, $37 \mu \mathrm{L}$ of APTES and $8 \mathrm{~mL}$ of FA $\left(1 \mathrm{~g} \mathrm{~L}^{-1}\right)$ were added to the solution, stirring for about $30 \mathrm{~min}$ to form the prepolymer complex, followed by addition of $50 \mu \mathrm{L}$ of ammonium hydroxide and $50 \mu \mathrm{L}$ of TEOS. The mixture was kept stirring for $12 \mathrm{~h}$ in the dark. Finally, the products were centrifuged and then eluted with methanol to remove FA template. The finally obtained particles were dispersed in $6 \mathrm{~mL}$ of ultrapure water to attain the FL-MIPs for further use. The flow chart for the preparation of FL-MIPs sensor was shown in Scheme S2. As a comparison, the non-imprinted polymers ratiometric fluorescence sensor (FLNIPs sensor, also NIPs for simplicity) were prepared under the same conditions but without adding FA. Related abbreviations were given in Table S2.

\subsection{Fluorescence measurement}

Before every measurement, the lamp intensity was calibrated by running a lamp scan and water scan (Fig. S2), as described in Experimental S4. For fluorescence measurement and FA visualization, $1.0 \mathrm{~mL}$ phosphate solution ( $\mathrm{pH}$ 7.5) containing $125 \mu \mathrm{L}$ of MIPs and different concentrations of FA from 0 to $5 \mathrm{mg} \mathrm{mL}^{-1}$ were reacted for $5 \mathrm{~min}$. All the fluorescence spectra were recorded under the same conditions: the slit widths of excitation and emission were both $5 \mathrm{~nm}$ and the excited light was set at $365 \mathrm{~nm}$ with an emission range of $400-700 \mathrm{~nm}$.

\subsection{Real sample analysis}

The practicality of the MIPs sensor was studied in several food samples, including four fresh vegetables and fruit (spinach, broccoli, tomatoes and oranges, purchased from a local supermarket (Nanchang, China)) which often contain natural FA, as well as FA-fortified milk powders (Yili group Co.Ltd, Inner Mongolia, China) and FA tablets (0.40 mg/tablet, Zhongzhou pharma Co. Ltd). These non-liquid real samples were pretreated into aqueous solutions before the fluorescence detection as given in Experimental S5. Then, for fluorescence detection using the developed FL-MIPs sensor, a proper volume of the sample solution (before and after spiked with FA at three concentrations of 10, 25 and $40 \mathrm{mg} \mathrm{L}^{-1}$ ) was mixed with the MIPs, with the final volume brought to $1 \mathrm{~mL}$ by readjusting phosphate buffer medium $(\mathrm{pH} 7.5$, $0.1 \mathrm{M})$. Specifically, the added volumes were 100, 100, 150, 320, 280, and $100 \mu \mathrm{L}$ for spinach, broccoli, tomato, orange, milk powder, and FA tablets, respectively. The $\mathrm{pH}$ values of the tested solutions were well controlled to be close to the optimized $\mathrm{pH}$ of 7.5, especially for the acidic tomato and orange samples: $\mathrm{pH}$ value was changed from original 4.21-7.45 for tomato, and changed from original 4.17-7.40 for orange.

Additionally, the FA contents in the aforementioned sample solutions were also tested by HPLC detection.

\section{Results and discussion}

\subsection{Preparation and characterization of FL-MIPs nanosensor}

Scheme 1 schematically illustrates the preparation process and visual principle of core-shell ratiometric fluorescence MIPs nanosensor. As seen, firstly, $\mathrm{SiO}_{2}$ nanoparticles were synthesized via a Stöber method to gain a core supporting material, which had many advantages of stable mechanical/chemical properties, good optical transparency, water solubility, low cost, easy preparation and good biocompatibility (Yan, He, Li, \& Zhang, 2017). Next, by virtue of the fluorescence of QDs and intrinsic luminescence of FA, the dual-emitting MIPs sensor excited with a signal wavelength in which this MIPs was assembled by one step with the assistance of the hydrolysis and condensation of APTES (functional monomer) and TEOS (cross-linker) in the presence of FA (template molecule), $\mathrm{NH}_{3} \cdot \mathrm{H}_{2} \mathrm{O}$ (catalyst), and CdTe QDs (signal recognition unit). After removing the embedded template FA, the core-shell MIPs sensor was prepared successfully. Hence, in contrast with traditional two-step procedure (Wang, Yu, Wu et al., 2016), this facile approach of one-step sol-gel polymerization greatly simplified the preparation procedure. The change in fluorescence characteristic depended on specific imprinting sites complementary in shape, size, and functional groups to FA (Scheme 1). As shown, the as-prepared MIPs only had the red-emission peak at $619 \mathrm{~nm}$ under $365 \mathrm{~nm}$ irradiation, stemming from the embedded QDs. After rebinding the template via the hydrogen bonding between the amino group of MIPs and the carboxyl group of FA, the red emissive QDs was quenched while the blue-emitting autofluorescence of FA appeared. Therefore, the MIPs presented two well-resolved emission peaks at 449 and $619 \mathrm{~nm}$, respectively, and displayed purple fluorescence under a UV lamp. Consequently, a remarkable color evolution from red to purple to final blue caused by the change in $\mathrm{I}_{449} / \mathrm{I}_{619}$ facilitated visualization of FA.

The morphological structures of $\mathrm{SiO}_{2}$, MIPs and NIPs were characterized by SEM and TEM. As shown in Fig. 1, it can be seen that the Stöber-prepared $\mathrm{SiO}_{2}$ nanoparticles were well dispersed with an average diameter of 75-85 nm; the MIPs displayed a uniform spherical structure with rough surface, verifying the formation of imprinted cavities on the surface of MIPs. The thicknesses of imprinted shell were estimated to be approximately 5-8 $\mathrm{nm}$ calculated from the TEM image (Fig. 1). Core-shell nanoscale structure with thin imprinting shell layer can ameliorate accessibility to binding site and facilitate mass transfer, enhancing the sensitivity to the template (Liu, Chen, Mu, Wang, \& Sun, 2016). Fig. S3 shows the size distribution of the above nanoparticles, i.e., the intensity contribution versus diameters, which was obtained by DLS measurement. As observed, the average size about $83 \mathrm{~nm}$ and $90 \mathrm{~nm}$ corresponded to $\mathrm{SiO}_{2}$ and FL-MIPs/NIPs, separately, which was in accordance with their TEM results and had a relatively identical size distribution with no remarkable aggregation. Obviously, above results testified that nanoscale core-shell-structured MIPs were successfully obtained.

To confirm the composition and atoms distribution of as-prepared MIPs, EDS analysis was clearly given in Fig. S4. In the spectrum of MIPs/NIPs, the existence of the peaks of $\mathrm{C}, \mathrm{O}, \mathrm{Si}, \mathrm{Cd}$, and Te elements substantiated successful construction of silica matrices and introduce of CdTe QDs in the imprinting matrix. While relatively low content of Te element made it only be detected weakly. Besides, no nitrogen peak was observed in MIPs and NIPs, possibly owing to that the nitrogen peak was covered by carbon peak close to it (Duan et al., 2014).

Fig. S5 presents the FT-IR spectra of $\mathrm{SiO}_{2}, \mathrm{MIPs}$, and NIPs. As seen, the characteristic peaks about $472,799 \mathrm{~cm}^{-1}$ was attributed to the symmetrical stretching vibration of Si-O, and the absorption peaks of Si$\mathrm{O}-\mathrm{Si}$ antisymmetric stretching were observed at about $1103 \mathrm{~cm}^{-1}$ with Si-OH bending vibration belonging to $979 \mathrm{~cm}^{-1}$, all of which indicated the successful introduction and presence of silica matrix in the two materials. The band around 1388 and $3452 \mathrm{~cm}^{-1}$ may coincide with the stretching band of the symmetric vibrations of $\mathrm{COO}^{-}$(Wang et al., 2018; Yu et al., 2012) and $\mathrm{N}-\mathrm{H}$, respectively, clearly confirming the existence of carboxyl groups in MPA-QDs and amino groups of functional monomer APTES, which revealed the successful preparation of FL-MIPs and FL-NIPs. 


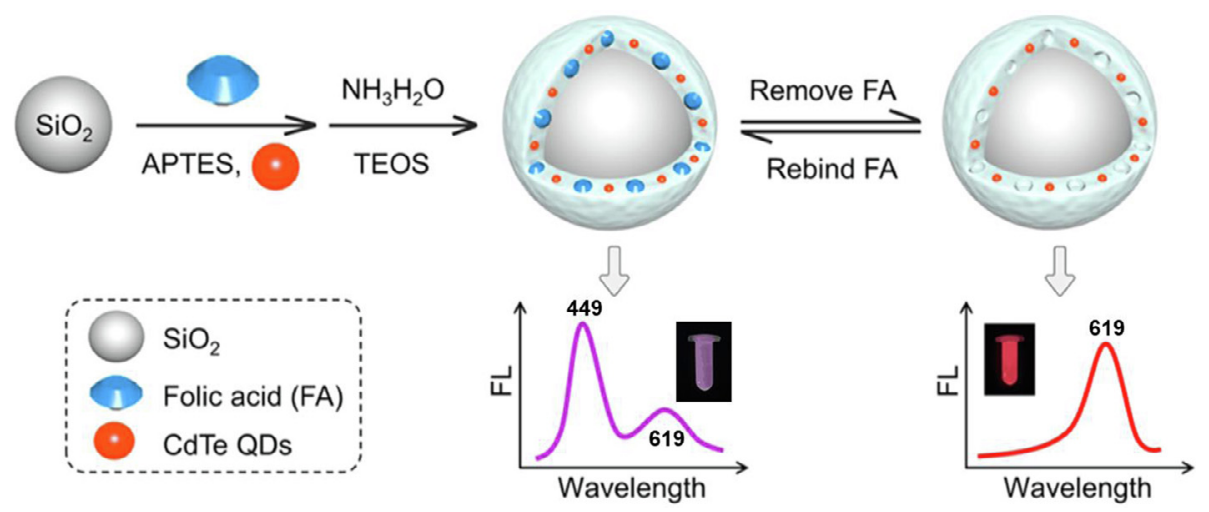

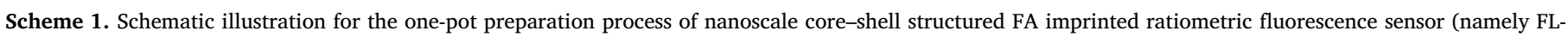
MIPs), and possible detection principle.

\subsection{Proposed detection mechanism of FL-MIPs nanosensor}

Generally, the mechanism of fluorescence quenching was either ascribed to electron transfer or energy transfer from CdTe@MIPs to analytes (Ensafi, Kazemifard, \& Rezaei 2017; Li, Liu, \& Ren, 2017). As revealed by the absorption spectrum of FA (curve a) and fluorescence emission spectrum of FL-MIPs (curve b) in Fig. S6, there was no spectral overlap between them, sequentially excluding the mechanism of energy transfer. Based on former studies (Ensafi, Nasr-Esfahani, \& Rezaei, 2017; Yang, Wang, Hui, \& Zhang, 2016), it was reasonable to speculate that the electron transfer originated from QDs and FA through strong interaction was responsible for the decrease of fluorescence intensity. The generated phenomenon was further explained based on electrontransfer induced fluorescence quenching, as depicted in Fig. S7a. A complex was formed between the carboxyl of FA and the amino group of FL-MIPs through the hydrogen bond forces. During the FA rebinding, the electron transfer between QDs and FA led to fluorescence quenching.

Meanwhile, Fig. S7b illustrates the mechanism via molecular orbital theory. In the absence of FA, the electron of QDs with accepting ultraviolet photon was excited from the valence band (ground-state) to the conduction band, successively, the excited electron got back to the ground-state, together with red fluorescence. With the existence of FA, the strong interaction force of hydrogen bond generated the shift of charge between QDs and FA. Besides, the ultraviolet absorption band of FA was very close to the band gap, which made the excited electron be prone to transition into the lowest unoccupied molecular orbital of the complex followed the paths shown as the dashed arrows. Therefore, no fluorescent signal appeared when the excited electron of QD return to the valence band once more, because the energy bands of the complex were higher than that of CdTe QDs. Ultimately, FA could be detected in this way.

\subsection{Variable optimization for the FL-MIPs sensor}

Several main variables which influenced FL-MIPs sensor efficiency were investigated, including the type of QDs, elution solvents, amount of TEOS, and the volume of MIPs.

Different types of quantum dots and various polar eluent solvents were considered all together. After our MIPs based on TGA-/MPA-/ GSH-modified QDs were eluted with methanol, acetonitrile (Acn) $/ \mathrm{H}_{2} \mathrm{O}$ $(\mathrm{v} / \mathrm{v}, 4: 1)$, ethanol/Acn $(\mathrm{v} / \mathrm{v}, 4: 1)$ and ethanol, respectively, the quenching rate on analyte $\mathrm{FA}$, defined as $F_{0} / F$, was demonstrated in Fig. S8. According to the Stern-Volmer equation $\left(F_{0} / F=1+\mathrm{Ksv}^{*}[C]\right.$, where $F_{0}$ and $F$ were the fluorescence intensities in the absence and presence of quencher, respectively, Ksv was the fluorescence quenching constant, $\mathrm{C}$ was the concentration of quencher), Ksv was selected as the evaluation indicator, which was positively correlated with quenching
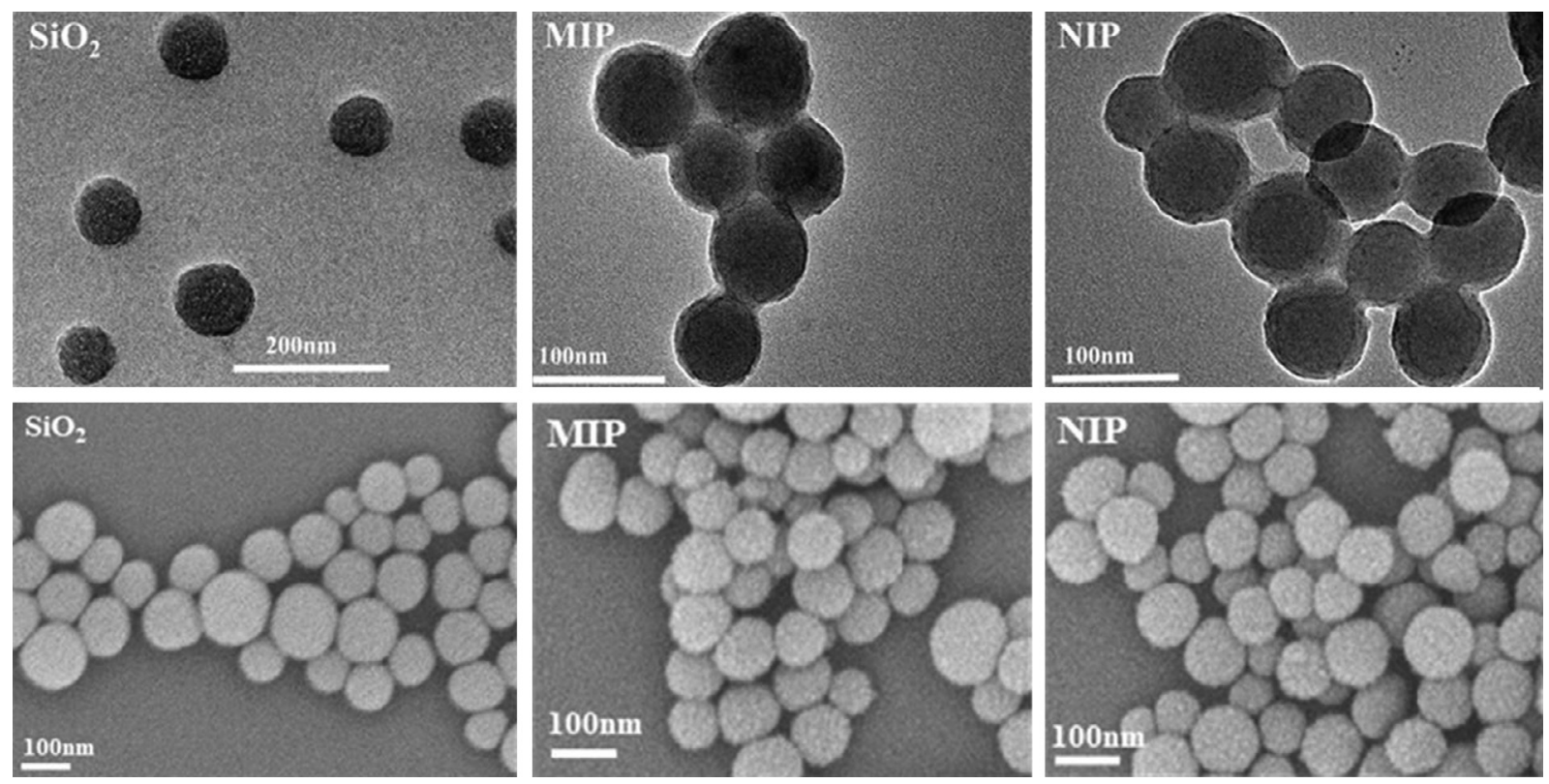

Fig. 1. TEM (upper) and SEM (below) images of $\mathrm{SiO}_{2}$, FL-MIPs and FL-NIPs. 
rate. In every type of QDs, Ksv value was maximum using methanol to remove the template. This may be due to that methanol was more liable to break the hydrogen bonds between APTES and trapped analyte FA. When methanol served as eluent, MPA-capped QDs always exhibited a superior value of Ksv. Comparison with the other two QDs, synthetic time of MPA-capped QDs was shorter and possessed better stability, no precipitation was observed when it left to $4{ }^{\circ} \mathrm{C}$ stored for more than three months. Finally, MPA-modified QDs was used in the preparation process of FL-MIPs sensor and the template was removed by methanol in order to attain maximal fluorescence-quenching efficiency.

The effect of the amount of cross-linking agent TEOS was investigated on fluorescence quenching. As can be seen from Fig. S9, the fluorescence quenching of FL-MIPs sensor declined gradually at a higher value than $50 \mu \mathrm{L}$. This phenomenon can be explained as: overmuch cross-linking between template and functional monomers lead that partial imprinting site deeply embedded into the polymer network, which hindered the removal and adsorption of the template. But, a lower degree of crosslinking was not conducive to the formation of the specific sites (Yan et al., 2017). Hence, $50 \mu \mathrm{L}$ of TEOS was adopted for the synthesis of FL-MIPs.

Fluorescence color evolution depending on the volume of MIPs played an essential role on visualization measurement, thus the response of varied amounts of FL-MIPs to a series of concentration FA (0-50 $\mathrm{mg} \mathrm{L}^{-1}$ ) was estimated. As seen from Fig. S10, when the volume of MIPs was $50 \mu \mathrm{L}$ and $100 \mu \mathrm{L}$, a majority of attended color was blue (Fig. S10A and B), because the strong fluorescence of FA masked the red fluorescence of QDs; besides, excessively low amounts of MIPs narrowed detection range. $125 \mu \mathrm{L}$ of the sensor was a better choice, exhibiting a more profuse color change successively in red, pink, purple and blue (Fig. S10C). However, at a volume $>125 \mu \mathrm{L}$, the red-emission of QDs was dominated over the fluorescence FA at excited light $365 \mathrm{~nm}$ (Fig. S10D and E), which caused that only obvious red and purple were observed, while blue hardly appearing. Therefore, $125 \mu \mathrm{L}$ of the MIPs could obtain the color-uniform variation for the naked-eye detection easily.

\subsection{Fluorescence properties of the FL-MIPs}

The relevant variables including $\mathrm{pH}$, incubation time and the fluorescence stability, which have a strong influence on analysis performances of the ratiometric FL-MIPs sensors for FA, were studied. The response of FL-MIPs to FA at $20 \mathrm{mg} \mathrm{L}^{-1}$ under different $\mathrm{pH}$ values was optimized in terms of the fluorescence intensity ratio, defined as $\mathrm{I}_{449}$ / $\mathrm{I}_{619}$. As shown in Fig. S11, it can be clearly seen that the fluorescence ratio increased with $\mathrm{pH}$ from 5 to 7.5 and then tended to be steady when $\mathrm{pH}$ values were higher. Because FA was not inclined to the acidic solution but depended on weak alkaline condition. So, the fluorescence intensity was relatively low below $\mathrm{pH} 7$ and the ratio reached a maximum level until $\mathrm{pH}$ 7.5. Additionally, the molecular-imprinted silica layers could be ionized under alkalinity $(\mathrm{pH}>9)$, which caused surface defect thereby affected the interaction between the FA and the fluorescence sensor. Therefore, $\mathrm{pH} 7.5$ was superior for the process of detection FA and a phosphate buffer solution ( $\mathrm{pH} 7.5)$ was chosen for all the following test.

The incubation time of the FL-MIPs sensor was also examined by monitoring the fluorescence recovery, and it acted as a function of time to evaluate the accessibility to the binding sites. As can be seen from Fig. 2, the fluorescence intensity at $619 \mathrm{~nm}$ decrease rapidly (had a cliff-type fall) upon the addition of FA at $20 \mathrm{mg} \mathrm{L}^{-1}$ within $1 \mathrm{~min}$ and subsequently, the decrement slowed down until the process reached equilibrium after $5 \mathrm{~min}$. The result was ascribed to the fact: in the beginning, due to the existence of many vacant imprinting cavities, rebinding and recognition of FA was easy to accomplish; As time extends, lots of sites were occupied and the discovery of suitable imprinted sites became hard for FA. In the inset, it was observed that fluorescence color of the probe solution immediately changed from red to purple. As

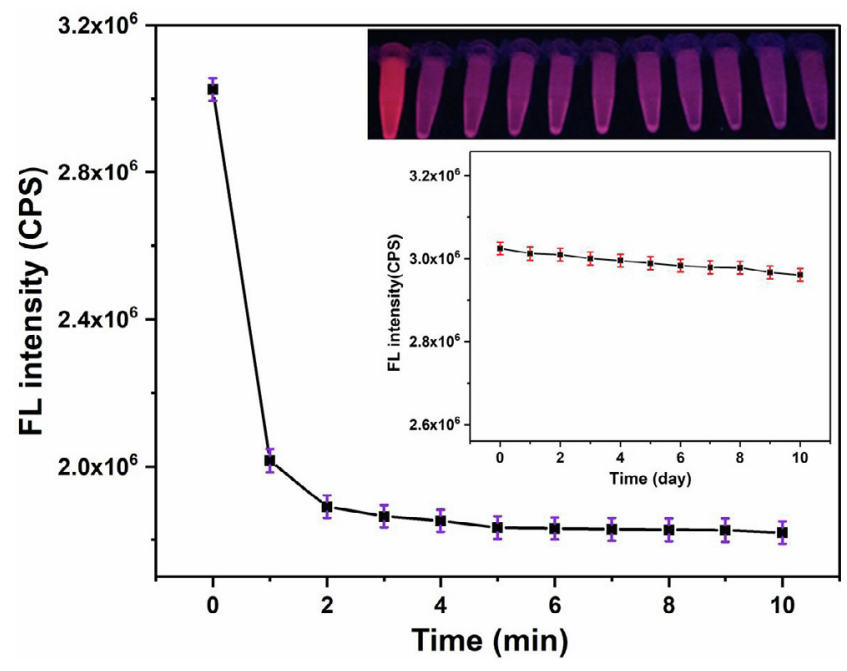

Fig. 2. Fluorescence response time of FL-MIPs snanosensor. The inset photography images in the upper right corner display the fluorescence colors under a $365 \mathrm{~nm}$ UV lamp from left to right corresponding to response time and the inset photo in the down is fluorescence stability of FL-MIPs. Experimental conditions: the concentration of FA at $20 \mathrm{mg} \mathrm{L}^{-1}$, the fluorescence intensity was recorded at the wavelength of $365 \mathrm{~nm}$.

results, $5 \mathrm{~min}$ was fixed as a proper time for the rebinding with high efficiency. The ultrathin imprinting layer on the surface of silica nanoparticle, which was seen from the TEM image of FL-MIPs with only several nanometers, provided more chance of being accessible to recognition site and achieved the fast response for targeted-analytes. Therefore, one of the effective ways to shorten incubation time was to reduce the imprinting-shell layer thickness (Wang et al., 2018).

Then, the fluorescence stability of FL-MIPs was examined via repeating the experiments of measuring fluorescence intensity at 10 a.m. every day during the storage (storage conditions: room temperature under subdued light). As shown in inset of Fig. 2, it was found that fluorescence intensity barely changed when the FL-MIPs were stored for 10 days, which certified that the resultant MIPs showed excellent physical and chemical stability.

\subsection{Sensitivity and selectivity of the FL-MIPs sensor}

Under the optimal conditions, the sensing performances of the FLMIPs involving sensitivity and selectivity were examined. As known to us, in the absence of FA, red fluorescence QDs at $619 \mathrm{~nm}$ could be just observed in principle when the excitation was at $365 \mathrm{~nm}$. But there was emerging weak peak at $449 \mathrm{~nm}$, which was in accordance with the inherent blue fluorescence peak of FA. This may be likely ascribed to not fully elution of the template molecules, nevertheless, it made no impact on target analysis in the testing process. As shown in Fig. 3A. With the FA increasing, the red fluorescence of QDs was quenched, while FA-trigger blue fluorescence enhanced. Based on the fluorescence emission intensity ratio, $\left(\mathrm{I}_{449} / \mathrm{I}_{619}\right) /\left(\mathrm{I}_{449} / \mathrm{I}_{619}\right)_{0}$, a standard linear plot was built for the quantification analysis of FA. From Fig. 3B, it was clearly observed, both polynomial fitting and linear fitting curve with $\mathrm{R}^{2}>0.99$ reflected an excellent linear relationship between the intensity ratio and the concentration of FA ranging from 0.1 to $50 \mathrm{mg} \mathrm{L}^{-1}$ $(0.23-113 \mu \mathrm{M})$. On the basis of $3 \sigma / s, \sigma / s$ namely the ratio of signal to noise, where $\sigma$ stands for the standard deviation of blank measurements (for 11 times), $s$ stands for the slope of the calibration curve) (Wang, Yu, Wu et al., 2016), the lowest detectable concentration, defined as limit of detection (LOD), was calculated to $48 \mathrm{nM}$, which made it become possible that low content of FA in real sample can be directly detected and the preenrichment processes in sample treatment were avoided. Benefit from the intensities change of two well-resolved emission peaks, 

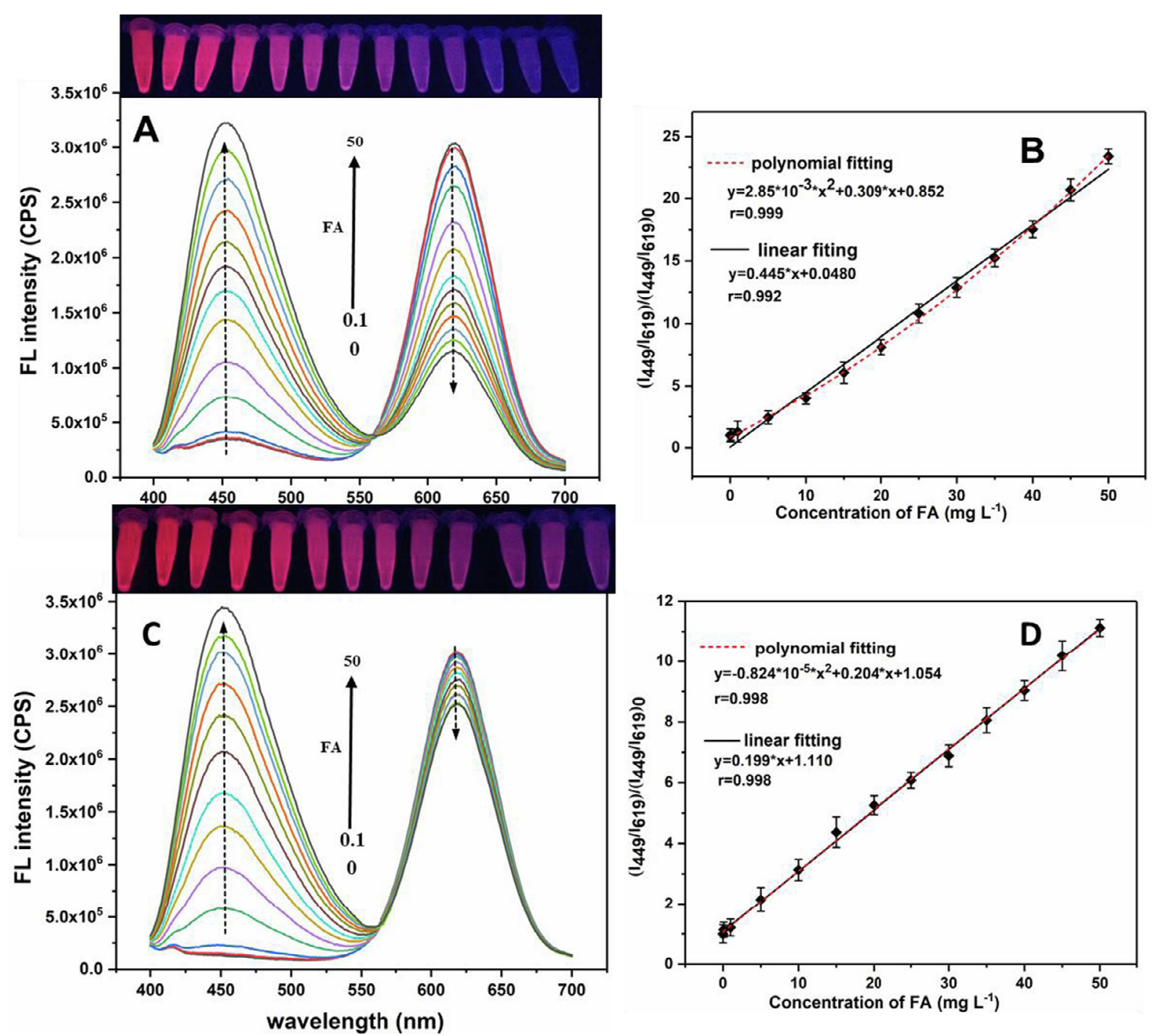

Fig. 3. Fluorescence emission spectra and fitting curves upon the exposure to FA concentrations from 0 to $50 \mathrm{mg} \mathrm{L}^{-1}$ for (A, B) FL-MIPs nanosensor and (C, D) FLNIPs nanosensor. The photography images in the upper (A, C) from left to right are their corresponding fluorescence color evolution taken under a $365 \mathrm{~nm}$ UV lamp. Experiment condition: excited wavelength: $365 \mathrm{~nm}$, silt widths of excitation and emission: $5 \mathrm{~nm}$.

a distinguishable color evolution from red to pink to purple to blue under a $365 \mathrm{~nm}$ UV lamp could be easily observed, as displayed in the upper of Fig. 3A, and it made feasible for detecting FA by the naked eyes.

In addition, the dosage response of NIPs to FA has been also examined to assess the recognition capability of imprinting memory and specific binding. From Fig. 3C, we found that red fluorescence of QDs was quenched slightly upon the addition of FA, which was attributed to the nonspecific recognition, and the absence of tailor-made binding sites complementary to template in size, shape and functional groups (Xu \& Lu, 2015), then an indistinctive difference among colors in NIPs was obtained (in the upper of Fig. 3C). The imprinting factor (IF) is the ratio of slope of the calibration curve for MIPs and NIPs, i.e. $\mathrm{K}_{\mathrm{SV}, \mathrm{MIP}}$ / $\mathrm{K}_{\mathrm{SV} \text {,NIP }}$ was used to appraise the difference in quenching efficiency between MIPs and NIPs, and IF value was counted to 2.24, which affirmed the recognition sites in FL-MIPs sensor were more and had a better steric matching ability towards FA, so more template molecules were allowed to enter into imprinted cavities. Hence, it could be reasonably inferred that MIPs based bimodal ratiometric fluorescence nanosensor had the capability of detecting FA with high precision, reliability and sensitivity.

Afterwards, the selectivity of MIPs and NIPs sensors was investigated. The fluorescence response (intensity ratio, $\left(\mathrm{I}_{449} / \mathrm{I}_{619}\right) /$ $\left.\left(\mathrm{I}_{449} / \mathrm{I}_{619}\right)_{0}\right)$ was investigated to diverse structural analogs, for instance MTX, TMP, Cys, His, Arg, Glu, and Vc (Fig. S1). As displayed in Fig. 4, the fluorescence quenching of FL-MIPs was approximately $40 \%$ when FA rebinding, which was higher than other compounds at the same concentration of $20 \mathrm{mg} \mathrm{L}^{-1}$. Admittedly, polymers generated the imprinted cavities complementary to the template in shape, size, and functional groups, in favor of specific recognition toward FA. It was worth noting that MIPs shown very low affinity toward other analogs, because of obvious distinctions among analogs and FA in terms of stereochemistry, size and functional groups, thereby fluorescent decline was primarily dominated by non-specific binding sites and was negligible. On the other side, the attention of a similar fluorescence ratio in NIPs sensor was paid to FA and its analogs, that was due to no recognition sites existence. Moreover, the color change of MIPs and NIPs solution was compared. Impressively, the MIPs went from original red to purple when FA rebind; nevertheless, keeping red color after the addition of other compounds (in the inset of Fig. 4). Naturally, the superiority of visualization for FA was present over other analogs. Logically, because of the lack of recognition sites in NIPs, both the control group of addition FA and the experimental group should keep consistent in color. But we observed that the former exhibited pink and the 


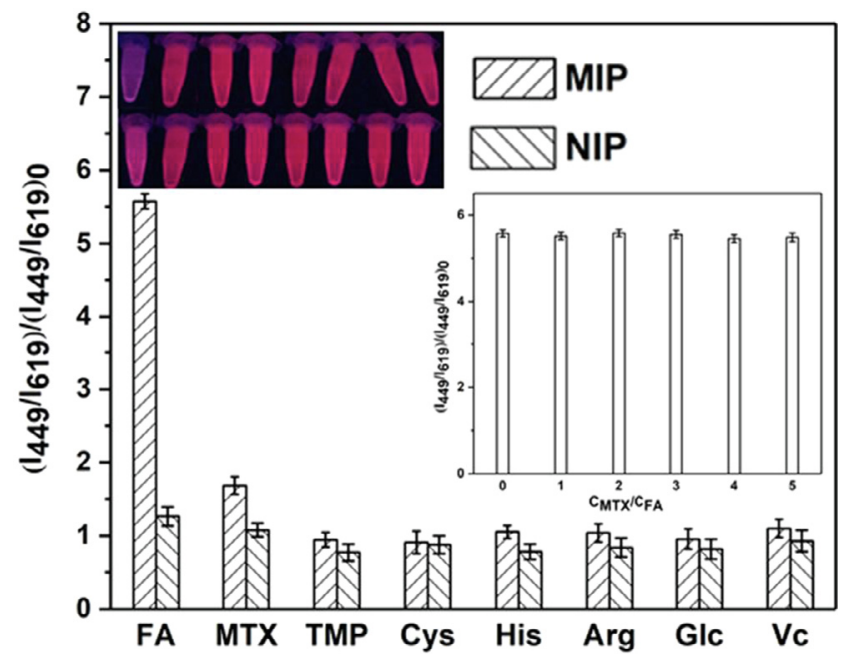

Fig. 4. Selectivity of MIPs and NIPs nanosensor for FA over other analogs including methotrexate (MTX), trimethoprim (TMP), cysteine (Cys), histidine (His), arginine (Arg), glutamic acid (Glu), and vitamin C (Vc) at the same concentration of $20 \mathrm{mg} \mathrm{L}^{-1}$. The photography images from left to right are sequentially their corresponding fluorescence color of the tested solution under a $365 \mathrm{~nm}$ UV lamp (MIPs in the upper, NIPs in the down). Inset: competitive test, the effect of the various concentration ratio between MTX and FA $\left(\mathrm{C}_{\mathrm{MTX}} /\right.$ $\left.\mathrm{C}_{\mathrm{FA}}\right)$ on the corresponding quenching amount $\left(I_{449} / I_{619}\right) /\left(I_{449} / I_{619}\right)_{0}$ for FLMIPs.

latter was red in Fig. 4. The blue-emitting fluorescence of FA was responsible for the difference between FA-NIPs solution and the experimental group in color. In the FA-NIPs solution, a certain amount mixture between red of QDs and intrinsic blue of template led to the emergence of pink but the other solution retain original red. In consequence, the above result further indicated that the FL-MIPs sensor had an advantage of higher selective capability and easy visual assessment toward FA.

Additionally, the results from competitive binding experiments, i.e. anti-interference trial of altering the proportion of MTX toward FA, were reported in Fig. 4 (insert). As can be reckoned from them, the quenching amount displayed no apparent change when increasing the ratio of $\mathrm{C}_{\mathrm{MTX}} / \mathrm{C}_{\mathrm{FA}}$. It should be adopted the fact that the steric hindrance of the methyl and amino groups in MTX structure with a bigger molecular weight than FA (Fig. S1), which was not beneficial to getting into the recognition site of FL-MIPs, and implied that the ability of the resistance of FL-MIPs to interference was considerably great. Both selectivity and competitive tests further confirmed that FL-MIPs had a better selectivity toward target molecule for optical determination.

\subsection{Applications of the nanosensor to real samples}

As for the practical application of proposed method in this study, six representative real samples were investigated by the methods and the traditional HPLC-UV. Fig. S12 shows that peak area change in HPLC with the concentration of FA, and Table 1 summarizes the corresponding analysis results. As listed above, in fortified foods, by the usage of our sensor, it was found that the contents of FA in the milk powder and FA tablets for pregnant women were about $228.2 \mu \mathrm{g} / 100 \mathrm{~g}$ and $0.37 \mathrm{mg} /$ slice, respectively, and conformed a criterion supplied by National Standard GB 14880-2012 and European Union Standard EC 1925-2006. The detection value was a bit lower than label indicated level of $2500 \mathrm{\mu g} \mathrm{kg}^{-1}$ and $0.40 \mathrm{mg} /$ slice, but within the allowable error range according to National Standard GB 28050-2011. In four foodstuffs (spinach, broccoli, tomato, and orange), the detected values by our sensor were within $265.8 \mu \mathrm{g} / 100 \mathrm{~g}, 180.9 \mu \mathrm{g} / 100 \mathrm{~g}, 84.2 \mu \mathrm{g} / 100 \mathrm{~g}$, and $37.4 \mu \mathrm{g} / 100 \mathrm{~g}$, respectively.

Furthermore, the recoveries for real samples were assessed by the determination of FA at three different spiked concentration (10, 25 and $40 \mathrm{mg} \mathrm{L}^{-1}$ ). As shown in Table 1 , the Relative standard deviations (RSDs) of $1.5-4.2 \%$ with favorable recoveries of $94.8-104.2 \%$ were obtained, which suggested the feasibility of the as-obtained sensor for selective, sensitive and accurate detection FA in samples. Moreover, the all results offered by our method were in good agreement with those validated by HPLC-UV, and possessed low relative errors of -14.28 to $8.33 \%$ in comparison with that of HPLC. The $t$-test $(\mathrm{P}>5 \%$ ) indicated that there was no significant difference between the two methods with 95\% confidence level.

\subsection{Method performance comparison}

In contrast with previously reported MIPs-based sensors, the analytical performance of our novel sensor was given in Table S1. As seen, it was noteworthy that we obtained a lower LOD in comparison with based-MIPs quartz crystal microbalance sensors (Apodaca, Pernites, Ponnapati, Del Mundo, \& Advincula, 2011; Hussain, Iqbal, \& Lieberzeit, 2013). More excitingly, a slightly lower detection concentration was observed than some electroanalysis methods (Karimian, Zavar, Chamsaz, Turner, \& Tiwari, 2013; Pereira, Cervini, \& Cavalheiro, 2014; Prasad, Madhuri, Tiwari, \& Sharma, 2010), which made the low-level FA can be sensitively recognized. And the wider linear range facilitated the FA detection in various practical samples, whether low or high concentration (Ensafi, Nasr-Esfahani, et al., 2017; Prasad, Tiwari, Madhuri, \& Sharma, 2010). As well as, ratiometric fluorescence system based on molecular imprinting was firstly used to determinate FA and provided a profuse color evolution according to the change in fluorescence intensities ratio, up to visualization effect. However, in other two florimetry methods, CdTe-labeled hydrophilic MIPs nanoparticles (Yang et al., 2016) and CdTe QD $_{\mathrm{o}}$-MIPs nanoparticles (Ensafi, NasrEsfahani, et al., 2017) only presented fluorescence brightness variation, i.e., yellow or orange gradually darkened, or even there were no color change in certain methods (Apodaca et al., 2011; Hussain et al., 2013; Karimian et al., 2013; Pereira et al., 2014; Prasad, Madhuri, et al., 2010; Prasad, Tiwari, et al., 2010). Hence, this work was promising in the future in terms of sensitivity, visualization, and practicability.

\section{Conclusions}

In summary, we firstly presented a nanoscale core-shell structured ratiometric fluorescence MIPs sensor for the specific identification and sensitive detection of FA. By integrating the superior sensitivity of fluorescence detection and excellent selectivity of MIPs, the sensor could specifically recognize and precisely quantify trace FA in complex matrices. Additionally, the sensor provided a low LOD and satisfactory recoveries. And for dual-emission ratiometric nanosensor, the timesaving one-pot construction strategy effectively avoided sophisticated multi-step preparation and simplified synthetic process. As well as, core-shell structure eased mass transfer resistance and provided more accessible binding sites to enable higher sensitivity. Both synthesis route and determination method possessed the impressive merits, involving facile generation, easy accessibility, low-cost, high efficiency, high accuracy, and reliability. As a successful proof-of-concept, this work holds much promise for promoting the rapid development of molecular imprinting ratiometric fluorescence sensors, and providing a universally applicable platform to serve for detection of other substances, e.g. other vitamins, micro elements or toxins in food products.

\section{Declaration of Competing Interest}

The authors declare that they have no known competing financial interests or personal relationships that could have appeared to influence the work reported in this paper. 
Table 1

Endogenous contents and spiked recoveries for the determination of FA in real samples by the developed FL-MIP nanosensor and conventional HPLC-UV $(n=3)$.

\begin{tabular}{|c|c|c|c|c|c|c|}
\hline \multirow[t]{2}{*}{ Sample } & \multirow[t]{2}{*}{ Spiked (mg/L) } & \multicolumn{2}{|c|}{ Found (mg/L) } & \multirow[t]{2}{*}{ Relative error (\%) against HPLC-UV } & \multicolumn{2}{|c|}{ Recovery $^{\mathrm{a}} \pm \mathrm{RSD}^{\mathrm{b}}(\%)$} \\
\hline & & MIP sensor & HPLC-UV & & MIP sensor & HPLC-UV \\
\hline \multirow[t]{4}{*}{ Milk powder } & 0 & 0.33 & 0.29 & 13.79 & - & - \\
\hline & 10 & 9.96 & 10.03 & -0.70 & $96.42 \pm 2.8$ & $97.47 \pm 2.5$ \\
\hline & 25 & 25.12 & 26.02 & -3.46 & $99.17 \pm 4.2$ & $102.89 \pm 3.1$ \\
\hline & 40 & 39.97 & 40.48 & -1.26 & $99.10 \pm 3.5$ & $100.47 \pm 2.9$ \\
\hline Tablets & 0 & 9.25 & 9.21 & 0.43 & - & - \\
\hline \multirow[t]{4}{*}{ Spinach } & 0 & 0.27 & 0.26 & 3.85 & - & - \\
\hline & 10 & 10.03 & 10.33 & -2.90 & $97.66 \pm 2.4$ & $100.68 \pm 3.4$ \\
\hline & 25 & 25.28 & 25.60 & -1.25 & $100.04 \pm 3.2$ & $101.35 \pm 2.6$ \\
\hline & 40 & 39.53 & 40.01 & -1.20 & $98.16 \pm 3.5$ & $99.38 \pm 3.5$ \\
\hline \multirow[t]{4}{*}{ Broccoli } & 0 & 0.18 & 0.18 & 0.00 & - & - \\
\hline & 10 & 9.65 & 9.86 & -2.13 & $94.79 \pm 2.6$ & $96.86 \pm 2.7$ \\
\hline & 25 & 25.59 & 25.05 & 2.16 & $101.63 \pm 3.5$ & $99.48 \pm 3.2$ \\
\hline & 40 & 40.05 & 40.17 & -0.30 & $99.68 \pm 3.1$ & $99.98 \pm 3.5$ \\
\hline \multirow[t]{4}{*}{ Tomato } & 0 & 0.13 & 0.12 & 8.33 & - & - \\
\hline & 10 & 10.05 & 9.88 & 1.72 & $99.21 \pm 2.8$ & $97.63 \pm 2.9$ \\
\hline & 25 & 24.68 & 25.41 & -2.87 & $98.21 \pm 2.5$ & $101.16 \pm 3.1$ \\
\hline & 40 & 40.57 & 39.74 & 2.09 & $101.10 \pm 3.1$ & $99.05 \pm 2.6$ \\
\hline \multirow[t]{4}{*}{ Orange } & 0 & 0.12 & 0.14 & -14.28 & - & - \\
\hline & 10 & 9.75 & 10.13 & -3.75 & $96.30 \pm 3.8$ & $99.97 \pm 4.2$ \\
\hline & 25 & 26.17 & 25.30 & 3.44 & $104.18 \pm 2.5$ & $100.60 \pm 2.7$ \\
\hline & 40 & 40.82 & 41.48 & -1.59 & $101.74 \pm 4.1$ & $103.33 \pm 2.9$ \\
\hline
\end{tabular}

a Average value from three individual experiments.

b Relative standard deviation.

\section{Acknowledgements}

This work was financially supported by the National Natural Science Foundation of China (21667018, 31660482, 31860470, 21804010, 21575159 and 21876199), the Chinese Academy of Sciences President's International Fellowship Initiative (2019PC0050) and the Taishan Scholar Project Special Funding, China (ts20190962).

\section{Appendix A. Supplementary data}

Supplementary data to this article can be found online at https:// doi.org/10.1016/j.foodchem.2020.126575.

\section{References}

Apodaca, D. C., Pernites, R. B., Ponnapati, R. R., Del Mundo, F. R., \& Advincula, R. C (2011). Electropolymerized molecularly imprinted polymer films of a bis-terthiophene dendron: Folic acid quartz crystal microbalance sensing. Acs Applied Materials \& Interfaces, 3(2), 191-203. https://doi.org/10.1021/ma2010525.

Chen, L., Xu, S., \& Li, J. (2011). Recent advances in molecular imprinting technology: Current status, challenges and highlighted applications. Chemical Society Reviews, 40(5), 2922-2942. https://doi.org/10.1039/C0CS00084A.

Chen, C., Luo, J., Li, C., Ma, M., Yu, W., Shen, J., \& Wang, Z. (2018). Molecularly imprinted polymer as an antibody substitution in pseudo-immunoassays for chemical contaminants in food and environmental samples. Journal of Agricultural and Food Chemistry, 66(11), 2561-2571. https://doi.org/10.1021/acs.jafc.7b05577.

Delchier, N., Herbig, A. L., Rychlik, M., \& Renard, C. M. (2016). Folates in fruits and vegetables: Contents, processing, and stability. Comprehensive Reviews in Food Science and Food Safety, 15(3), 506-528. doi: 10.1111/1541-4337.12193.

Duan, F., Chen, C., Lin, C., Sun, Y., Wang, Y., Yang, Y., ... Yong, Q. (2014). Preparation and evaluation of water-compatible surface molecularly imprinted polymers for selective adsorption of bisphenol a from aqueous solution. Industrial and Engineering Chemistry Research, 53(37), 14291-14300. https://doi.org/10.1021/ie5028099.

Ensafi, A. A., Kazemifard, N., \& Rezaei, B. (2017). Development of a selective prilocaine optical sensor based on molecularly imprinted shell on CdTe quantum dots. Sensors and Actuators B:Chemical, 242, 835-841. https://doi.org/10.1016/j.snb.2016.09.175.

Ensafi, A. A., Nasr-Esfahani, P., \& Rezaei, B. (2017). Simultaneous detection of folic acid and methotrexate by an optical sensor based on molecularly imprinted polymers on dual-color CdTe quantum dots. Analytica Chimica Acta, 996, 64-73. https://doi.org/ 10.1016/j.aca.2017.10.011.

Fu, Y., Jin, H., Bu, X., \& Gui, R. (2018). Melamine-induced decomposition and anti-FRET effect from self-assembled complex of rhodamine $6 \mathrm{G}$ and DNA-stabilized silver nanoclusters used for dual-emitting ratiometric and naked-eye visual fluorescence detection. Journal of Agricultural and Food Chemistry, 66(37), 9819-9827. https://doi org/10.1021/acs.jafc.8b03402.

Hemmateenejad, B., Shakerizadeh-shirazi, F., \& Samari, F. (2014). BSA-modified gold nanoclusters for sensing of folic acid. Sensors and Actuators B: Chemical, 199, 42-46. https://doi.org/10.1016/j.snb.2014.03.075.

Hussain, M., Iqbal, N., \& Lieberzeit, P. A. (2013). Acidic and basic polymers for molecularly imprinted folic acid sensors-QCM studies with thin films and nanoparticles. Sensors and Actuators B: Chemical, 176, 1090-1095. https://doi.org/10.1016/j.snb. 2012.09.082.

Karimian, N., Zavar, M. H. A., Chamsaz, M., Turner, A. P. F., \& Tiwari, A. (2013). On/offswitchable electrochemical folic acid sensor based on molecularly imprinted polymer electrode. Electrochemistry Communications, 36(6), 92-95. https://doi.org/10.1016/j. elecom.2013.09.014.

Komasawa, N., Ueki, R., Kaminoh, Y., \& Nishi, S. I. (2010). Comparison of QuEChERS sample preparation methods for the analysis of pesticide residues in fruits and vegetables. Journal of Chromatography A, 1217(16), 2548-2560. https://doi.org/10. 1016/j.chroma.2010.01.044.

Leporati, A., Catellani, D., Suman, M., Andreoli, R., Manini, P., \& Niessen, W. M. (2005). Application of a liquid chromatography tandem mass spectrometry method to the analysis of water-soluble vitamins in Italian pasta. Analytica Chimica Acta, 531, 87-95. https://doi.org/10.1016/j.aca.2004.10.006.

Li, J., Fu, J., Yang, Q., Wang, L., Wang, X., \& Chen, L. (2018). Thermosensitive molecularly imprinted core-shell CdTe quantum dots as a ratiometric fluorescence nanosensor for phycocyanin recognition and detection in seawater. Analyst, 143(15), 3570-3578. https://doi.org/10.1039/C8AN00811F.

Li, M., Liu, H., \& Ren, X. (2017). Ratiometric fluorescence and mesoporous structured imprinting nanoparticles for rapid and sensitive detection 2,4,6-trinitrophenol. Biosensors and Bioelectronics, 89, 899-905. https://doi.org/10.1016/j.bios.2016.09. 101.

Li, W., Zhang, H., Chen, S., Liu, Y., Zhuang, J., \& Lei, B. (2016). Synthesis of molecularly imprinted carbon dot grafted YVO4:Eu3 + for the ratiometric fluorescent determination of paranitrophenol. Biosensors and Bioelectronics, 86, 706-713. https://doi. org/10.1016/j.bios.2016.07.034.

Li, Z., Gueant-Rodriguez, R. M., Quilliot, D., Sirveaux, M. A., Meyre, D., Gueant, J. L., \& Brunaud, L. (2017). Folate and vitamin B12 status is associated with insulin resistance and metabolic syndrome in morbid obesity. Clinical Nutrition, 37(5), 1700-1706. https://doi.org/10.1016/j.clnu.2017.07.008.

Liu, H., Chen, X., Mu, L., Wang, J., \& Sun, B. (2016). Application of quantum dot-molecularly imprinted polymer core-shell particles sensitized with graphene for Optosensing of $\mathrm{N} \varepsilon$-Carboxymethyllysine in dairy products. Journal of Agricultural and Food Chemistry, 64(23), 4801-4806. https://doi.org/10.1021/acs.jafc.6b01504.

Menger, M., Yarman, A., Erdossy, J., Yildiz, H. B., Gyurcsanyi, R. E., \& Scheller, F. W. (2016). MIPs and aptamers for recognition of proteins in biomimetic sensing. Biosensors, 6(3), 35. https://doi.org/10.3390/bios6030035.

Monica, A. F., Alain, G., Elodie, R., \& Laurent, B. (2014). Rapid HPLC-MS method for the simultaneous determination of tea catechins and folates. Journal of Agricultural and Food Chemistry, 62(19), 4241-4250. https://doi.org/10.1021/jf4053258.

Pawlosky, R. J., \& Flanagan, V. P. (2001). A quantitative stable-isotope LC-MS method for the determination of folic acid in fortified foods. Journal of Agricultural and Food 
Chemistry, 49(3), 1282-1286. https://doi.org/10.1021/jf001172i.

Pereira, A. V., Cervini, P., \& Cavalheiro, É. T. G. (2014). Do voltammetry electrodes modified with MIPs really work? The role of large molecules: Folic acid as a probe. Analytical Methods, 6(17), 6658-6667. https://doi.org/10.1039/C4AY00616J.

Prasad, B. B., Tiwari, M. P., Madhuri, R., \& Sharma, P. S. (2010). a). Development of a highly sensitive and selective hyphenated technique (molecularly imprinted microsolid phase extraction fiber-molecularly imprinted polymer fiber sensor) for ultratrace analysis of folic acid. Analytica Chimica Acta, 662, 14-22. https://doi.org/10. 1016/j.aca.2009.12.037.

Prasad, B. B., Madhuri, R., Tiwari, M. P., \& Sharma, P. S. (2010). b). Electrochemical sensor for folic acid based on a hyperbranched molecularly imprinted polymer-immobilized sol-gel-modified pencil graphite electrode. Sensors and Actuators B: Chemical, 146, 321-330. https://doi.org/10.1016/j.snb.2010.02.025.

Qi, J., Li, B. W., Wang, X. R., Zhang, Z., Wang, Z., Han, J. L., \& Chen, L. X. (2017). Threedimensional paper-based microfluidic chip device for multiplexed fluorescence detection of $\mathrm{Cu}^{2+}$ and $\mathrm{Hg}^{2+}$ ions based on ion imprinting technology. Sensors and Actuators B: Chemical, 251, 224-233. https://doi.org/10.1016/j.snb.2017.05.052.

Vishnumohan, S., Arcot, J., \& Pickford, R. (2011). Naturally-occurring folates in foods: Method development and analysis using liquid chromatography-tandem mass spectrometry (LC-MS/MS). Food Chemistry, 125, 736-742. https://doi.org/10.1016/j. foodchem.2010.08.032.

Wang, H., De, S. H., Chen, G., Zhang, X., Pei, L., Gellynck, X., \& Zheng, X. (2016). Effectiveness of folic acid fortified flour for prevention of neural tube defects in a high risk region. Nutrients, 8(3), 152. https://doi.org/10.3390/nu8030152.

Wang, J., Hao, Y., Wang, H., Yang, S., Tian, H. Y., Sun, B., \& Liu, Y. (2017). Rapidly responsive and highly selective fluorescent probe for bisulfite detection in food. Journal of Agricultural and Food Chemistry, 65(13), 2883-2887. https://doi.org/10. 1021/acs.jafc.7b00353.

Wang, J., Wang, H., Hao, Y., Yang, S., Tian, H., Sun, B., \& Liu, Y. (2018). A novel reactionbased fluorescent probe for the detection of cysteine in milk and water samples. Food Chemistry, 262, 67-71. https://doi.org/10.1016/j.foodchem.2018.04.084.

Wang, X., Yu, J., Kang, Q., Shen, D., Li, J., \& Chen, L. (2016). Molecular imprinting ratiometric fluorescence sensor for highly selective and sensitive detection of phycocyanin. Biosensors and Bioelectronics, 77, 624-630. https://doi.org/10.1016/j.bios. 2015.10.019.

Wang, X., Yu, J., Wu, X., Fu, J., Kang, Q., Shen, D., ... Chen, L. (2016). A molecular imprinting-based turn-on Ratiometric fluorescence sensor for highly selective and sensitive detection of 2,4-dichlorophenoxyacetic acid (2,4-D). Biosensors and Bioelectronics, 81, 438-444. https://doi.org/10.1016/j.bios.2016.03.031.

Wang, X., Yu, S., Liu, W., Fu, L., Wang, Y., Li, J., \& Chen, L. (2018). A molecular imprinting based hybrid ratiometric fluorescence sensor for the visual determination of bovine hemoglobin. ACS Sensors, 3(2), 378-385. https://doi.org/10.1021/ acssensors.7b00804.

Wu, L., Lin, Z. Z., Zhong, H. P., Peng, A. H., Chen, X. M., \& Huang, Z. Y. (2017). Rapid detection of malachite green in fish based on CdTe quantum dots coated with molecularly imprinted silica. Food Chemistry, 229, 847-853. https://doi.org/10.1016/j. foodchem.2017.02.144.

Wu, X., Zhang, Z., Li, J., You, H., Li, Y., \& Chen, L. (2015). Molecularly imprinted polymers-coated gold nanoclusters for fluorescent detection of bisphenol A. Sensors and Actuators B: Chemical, 211, 507-514. https://doi.org/10.1016/j.snb.2015.01. 115.

$\mathrm{Xu}, \mathrm{S}$., \& Lu, H. (2015). One-pot synthesis of mesoporous structured ratiometric fluorescence molecularly imprinted sensor for highly sensitive detection of melamine from milk samples. Biosensors and Bioelectronics, 73, 160-166. https://doi.org/10.1016/j. bios.2015.05.064.

Xu, S., Lu, H., Li, J., Song, X., Wang, A., Chen, L., \& Han, S. (2013). Dummy molecularly imprinted polymers-capped CdTe quantum dots for the fluorescent sensing of 2,4,6 trinitrotoluene. Applied Materials and Interfaces, 5(16), 8146-8154. https://doi.org/ 10.1021/am4022076.

Yan, Y.-J., He, X.-W., Li, W.-Y., \& Zhang, Y.-K. (2017). Nitrogen-doped graphene quantum dots-labeled epitope imprinted polymer with double templates via the metal chelation for specific recognition of cytochrome c. Biosensors and Bioelectronics, 91, 253-261. https://doi.org/10.1016/j.bios.2016.12.040.

Yang, Q., Li, J., Wang, X., Peng, H., Xiong, H., \& Chen, L. (2018). Strategies of molecular imprinting-based fluorescence sensors for chemical and biological analysis. Biosensors and Bioelectronics, 112, 54-71. https://doi.org/10.1016/j.bios.2018.04.028.

Yang, Y., Wang, Z., Hui, N., \& Zhang, H. (2016). One-pot synthesis of quantum dotlabeled hydrophilic molecularly imprinted polymer nanoparticles for direct optosensing of folic acid in real, undiluted biological samples. Biosensors and Bioelectronics, 86, 580-587. https://doi.org/10.1016/j.bios.2016.07.056.

Yang, Y. Q., He, X. W., Wang, Y. Z., Li, W. Y., \& Zhang, Y. K. (2014). Epitope imprinted polymer coating CdTe quantum dots for specific recognition and direct fluorescent quantification of the target protein bovine serum albumin. Biosensors and Bioelectronics, 54, 266-272. https://doi.org/10.1016/j.bios.2013.11.004.

Yu, Y., Xu, L., Chen, J., Gao, H., Wang, S., Fang, J., \& Xu, S. (2012). Hydrothermal synthesis of GSH-TGA co-capped CdTe quantum dots and their application in labeling colorectal cancer cells. Colloids and Surfaces B-Biointerfaces, 95, 247-253. https://doi. org/10.1016/j.colsurfb.2012.03.011.

Zhang, W., Han, Y., Chen, X., Luo, X., Wang, J., Yue, T., \& Li, Z. (2017). Surface molecularly imprinted polymer capped Mn-doped ZnS quantum dots as a phosphorescent nanosensor for detecting patulin in apple juice. Food Chemistry, 232, 145-154. https://doi.org/10.1016/j.foodchem.2017.03.156. 